NASA/CR-2005-213796

\title{
The Effect of Pulse Length and Ejector Radius on Unsteady Ejector Performance
}

Jack Wilson

QSS Group, Inc., Cleveland, Ohio 
Since its founding, NASA has been dedicated to the advancement of aeronautics and space science. The NASA Scientific and Technical Information (STI) Program Office plays a key part in helping NASA maintain this important role.

The NASA STI Program Office is operated by Langley Research Center, the Lead Center for NASA's scientific and technical information. The NASA STI Program Office provides access to the NASA STI Database, the largest collection of aeronautical and space science STI in the world. The Program Office is also NASA's institutional mechanism for disseminating the results of its research and development activities. These results are published by NASA in the NASA STI Report Series, which includes the following report types:

- $\quad$ TECHNICAL PUBLICATION. Reports of completed research or a major significant phase of research that present the results of NASA programs and include extensive data or theoretical analysis. Includes compilations of significant scientific and technical data and information deemed to be of continuing reference value. NASA's counterpart of peerreviewed formal professional papers but has less stringent limitations on manuscript length and extent of graphic presentations.

- TECHNICAL MEMORANDUM. Scientific and technical findings that are preliminary or of specialized interest, e.g., quick release reports, working papers, and bibliographies that contain minimal annotation. Does not contain extensive analysis.

- CONTRACTOR REPORT. Scientific and technical findings by NASA-sponsored contractors and grantees.
- CONFERENCE PUBLICATION. Collected papers from scientific and technical conferences, symposia, seminars, or other meetings sponsored or cosponsored by NASA.

- SPECIAL PUBLICATION. Scientific, technical, or historical information from NASA programs, projects, and missions, often concerned with subjects having substantial public interest.

- TECHNICAL TRANSLATION. Englishlanguage translations of foreign scientific and technical material pertinent to NASA's mission.

Specialized services that complement the STI Program Office's diverse offerings include creating custom thesauri, building customized databases, organizing and publishing research results ... even providing videos.

For more information about the NASA STI Program Office, see the following:

- Access the NASA STI Program Home Page at http://www.sti.nasa.gov

- E-mail your question via the Internet to help@sti.nasa.gov

- Fax your question to the NASA Access Help Desk at 301-621-0134

- Telephone the NASA Access Help Desk at 301-621-0390

- Write to:

NASA Access Help Desk

NASA Center for AeroSpace Information 7121 Standard Drive

Hanover, MD 21076 
NASA/CR-2005-213796

AIAA-2005-3829

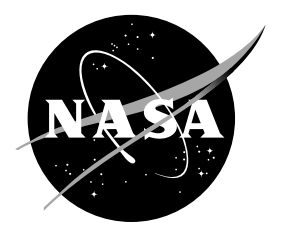

\section{The Effect of Pulse Length and Ejector Radius on Unsteady Ejector Performance}

Jack Wilson

QSS Group, Inc., Cleveland, Ohio

Prepared for the

41st Joint Propulsion Conference and Exhibit

cosponsored by the AIAA, ASME, SAE, and ASEE

Tucson, Arizona, July 10-13, 2005

Prepared under Contract NAS3-00145

National Aeronautics and

Space Administration

Glenn Research Center 


\section{Acknowledgments}

The author wishes to thank Robert Pastel and Kevin Dougherty for assistance with the experiments.

The support of the Constant Volume Combustion Cycle Engine (CVCCE) project, directed by Leo Burkardt, is acknowledged with gratitude.

This work was sponsored by the Low Emissions Alternative

Power Project of the Vehicle Systems Program at the NASA Glenn Research Center.

Available from

NASA Center for Aerospace Information 7121 Standard Drive

Hanover, MD 21076
National Technical Information Service 5285 Port Royal Road Springfield, VA 22100 


\title{
The Effect of Pulse Length and Ejector Radius on Unsteady Ejector Performance
}

\author{
Jack Wilson \\ QSS Group, Inc, \\ Cleveland, Ohio 44135
}

\begin{abstract}
The thrust augmentation of a set of ejectors driven by a shrouded Hartmann-Sprenger tube has been measured at four different frequencies. Each frequency corresponded to a different length to diameter ratio of the pulse of air leaving the driver shroud. Two of the frequencies had length to diameter ratios below the formation number, and two above. The formation number is the value of length to diameter ratio below which the pulse converts to a vortex ring only, and above which the pulse becomes a vortex ring plus a trailing jet.

A three level, three parameter Box-Behnken statistical design of experiment scheme was performed at each frequency, measuring the thrust augmentation generated by the appropriate ejectors from the set. The three parameters were ejector length, radius, and inlet radius.

The results showed that there is an optimum ejector radius and length at each frequency. Using a polynomial fit to the data, the results were interpolated to different ejector radii and pulse length to diameter ratios. This showed that a peak in thrust augmentation occurs when the pulse length to diameter ratio equals the formation number, and that the optimum ejector radius is 0.87 times the sum of the vortex ring radius and the core radius.
\end{abstract}

\section{Nomenclature}

a

$A_{\text {pulse }}$

$D_{\text {pulse }}$

$f$

K

$L_{e j}$

$L_{\text {pulse }}$

$\dot{m}_{\text {jet }}$

$m_{\text {pulse }}$

$\dot{m}_{\text {total }}$

$N$

$p$

$R$

$R_{e j}$

$R_{\text {exit }}$

$R_{S}$

$r_{n}$

$S_{t}$

$t$

$t^{\prime}$

$T_{\text {jet }}$

$u(t)$

$U$

$V_{\text {exit }}$

$\alpha$

$\alpha_{s s}$ radius of vortex ring core

exit area of the driving jet

$2 \sqrt{A_{\text {pulse }} / \pi}(44 \mathrm{~mm})$

frequency of driving jet

circulation in vortex ring

length of the ejector

length of the slug of air in each pulse

mass flow rate of the jet

mass of air in each pulse

total mass flow rate leaving the ejector

formation number

fraction of total jet momentum in the vortex ring

radius of the vortex ring

radius of the ejector

exit radius of the ejector

inner radius of the shroud $(25.4 \mathrm{~mm})$

ejector inlet radius

Strouhal Number $\left(f D_{\text {pulse }} / U\right)$

time

time at which the pulse ratio $L_{\text {pulse }} / D_{\text {pulse }}=N$

thrust of jet alone

jet velocity as a function of time

average velocity of the driving jet $=T_{\text {jet }} / \dot{m}_{\text {jet }}$

average in time and space of ejector exit velocity

thrust augmentation coefficient

thrust augmentation for $L_{\text {pulse }} / D_{\text {pulse }}=\infty$ 


$$
\begin{array}{ll}
\alpha_{t o t a l} & \text { weighted sum of } \alpha_{s s} \text { and } \alpha_{v r} \\
\alpha_{v r} & \text { thrust augmentation due to vortex ring only } \\
\left.\alpha_{v r}\right|_{N} & \alpha_{v r} \text { for } L_{\text {pulse }} / D_{\text {pulse }}=N \\
\beta & \text { mass entrainment coefficient }=\left(\dot{m}_{\text {total }}-\dot{m}_{\text {jet }}\right) / \dot{m}_{\text {jet }} \\
\varepsilon & a / R \\
\xi & \dot{m}_{\text {jet }} / \rho U A_{\text {pulse }} \\
\rho & \text { atmospheric air density } \\
\tau & \text { period of one pulse }=1 / f
\end{array}
$$

\section{Introduction}

The current interest in Pulsed Detonation Engines has revived interest in pulsed ejectors (refs. 1 to 3), since the latter can increase the thrust, and potentially reduce the noise level, of such engines with little additional hardware. There have been measurements on pulsed ejector performance in the past, using drivers that were not detonation devices. Lockwood (ref. 4), using a pulsejet driver, measured thrust augmentations as high as 1.9, with a very short ejector. Didelle (ref. 5), using a jet interrupted by a butterfly valve, also observed a maximum thrust augmentation ratio of 1.9, but with a much longer ejector. Bertin (ref. 6), also claimed a thrust augmentation ratio of around 2, but gave few details. More recently, Paxson, Wilson, and Dougherty (ref. 7) have repeated Lockwood's experiment, with similar results, although the optimum ejector was rather longer than was Lockwood's. Wilson and Paxson (ref. 8), using a Hartmann-Sprenger, or resonance, tube to generate a pulsed jet, measured a maximum thrust augmentation of 1.32, and found, as did Paxson, Wilson and Dougherty, that there was an optimum radius for the ejector. Despite these efforts, it is still not clear what are the important parameters for optimizing a pulsed ejector. Except for that of Didelle, in none of the above experiments was the length of the pulse driving the ejector changed. Didelle, who varied the jet frequency, and hence pulse length, found his maximum thrust augmentation at a Strouhal number of 0.07 .

A major feature of any pulsed jet flow is the starting vortex ring (Elder and deHaas (ref. 9), Das, Arekeri, Krothapalli, and Lourenco (ref. 10)). Gharib, Rambod, and Shariff (ref. 11) have shown that there is a critical value of the pulse length to diameter ratio $L_{\text {pulse }} / D_{\text {pulse, }}$ which they called the formation number $N$ : for values of $L_{p u l s e} / D_{\text {pulse }}<N$, the pulse transforms into a vortex ring only, for $L_{\text {pulse }} / D_{\text {pulse }}>N$, the flow becomes a vortex ring followed by a trailing jet. Since the structure of the flow changes in this way with $L_{p u l s e} / D_{p u l s e}$, one would expect that changing $L_{\text {pulse }} / D_{\text {pulse }}$ might also affect ejector performance.

In order to explore this possibility, experiments to measure pulsed thrust augmentation were made using a shrouded Hartmann-Sprenger tube as the pulsed source. This source, previously used by Wilson and Paxson (ref. 8), has the advantage that by varying the tube and shroud lengths, different frequencies can be generated, corresponding to different values of the pulse length to diameter ratio. A statistical experiment was designed to explore the effects of ejector length, radius, and nose radius. This was repeated at each of four frequencies, and by fitting the data with a two parameter model, the effect of $L_{p u l s e} / D_{p u l s e}$ and ejector radius on the thrust augmentation was determined. The maximum thrust augmentation was found to be at an $L_{p u l s e} / D_{\text {pulse }}$ equal to the formation number, and the optimum ejector radius was found to be slightly smaller than the size of the vortex ring, defined as $(R+a)$.

\section{Apparatus}

A photograph of the apparatus is shown in figure 1. Consisting of a shrouded Hartmann-Sprenger tube, a thrust plate, and a set of ejectors, it is the same as described by Wilson and Paxson (ref. 8), where a more detailed description will be found. The Hartmann-Sprenger tube (fig. 2) is a device which converts a steady, supersonic flow into a pulsed flow, by directing it into a resonance tube. It was shrouded to channel the pulsed flow into the axial direction. As initially built, the Hartmann-Sprenger tube operated at a frequency of $550 \mathrm{~Hz}$. Different frequencies were obtained by, first, putting a plug inside the resonance tube to shorten it, which produced a frequency of $1100 \mathrm{~Hz}$, and then making two longer tubes and shrouds to replace the original tube, to give frequencies of 275 and $125 \mathrm{~Hz}$. Since the nozzle upstream of the resonance tube is choked, the mass flow remained the same for all frequencies.

The set of ejectors is also given in figure 2, to illustrate the geometry. The set includes four different ejector radii, $R_{e j}$, at each of which three lengths, $L_{e j}$, are possible, as well as three inlet radii, $r_{n}$. Since there was a diffuser on each ejector, the exit radius is greater than the ejector radius itself. 
In running the experiment, the thrust of the jet without an ejector was measured, followed by the thrust with an ejector. The ratio of the two thrusts is the thrust augmentation $\alpha$. The exact experimental procedure is described in Wilson and Paxson (ref. 8).

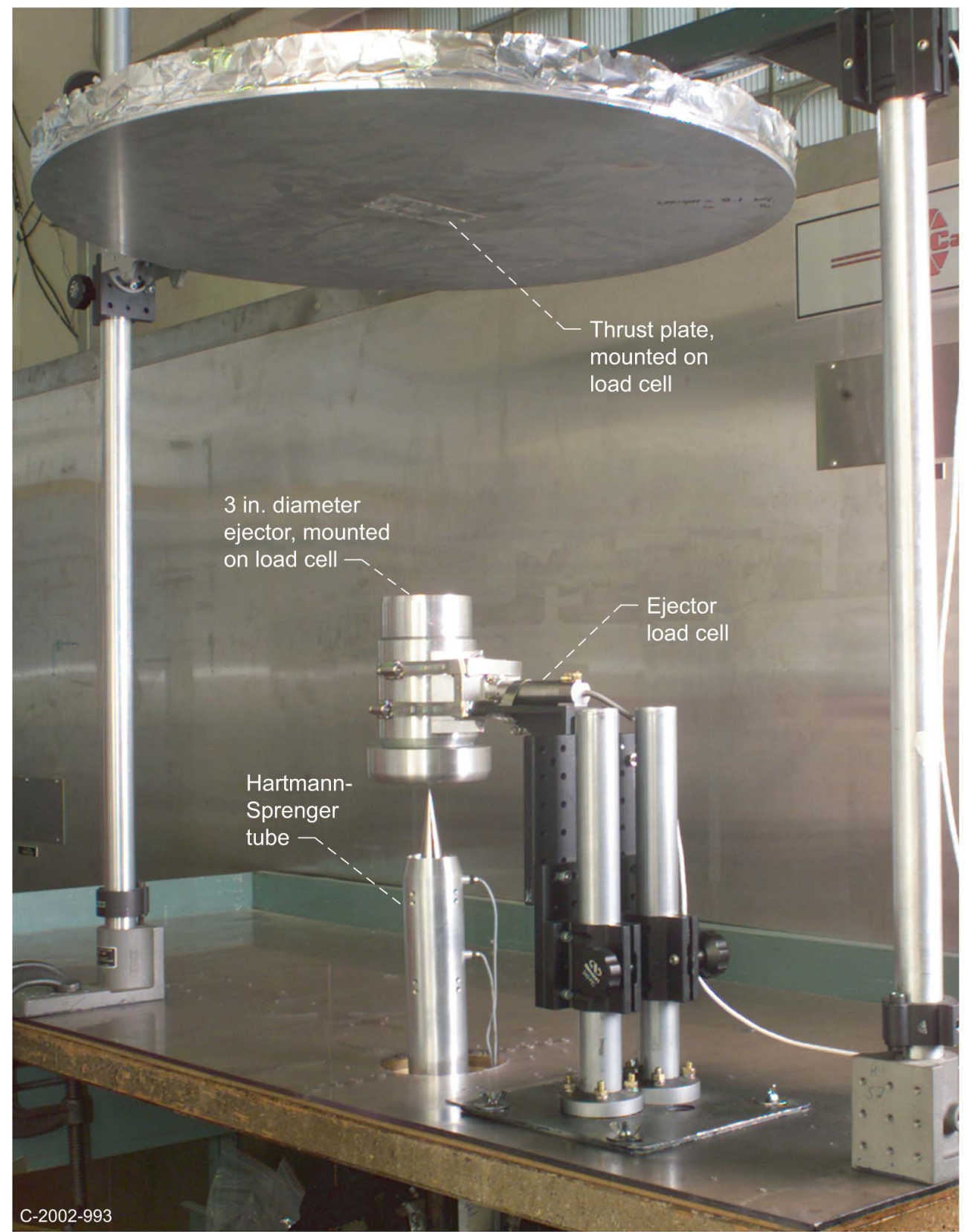

Figure 1. Photograph of the apparatus, showing the thrust plate at the top, the shrouded Hartmann-Sprenger tube at the bottom, and an ejector above it. 

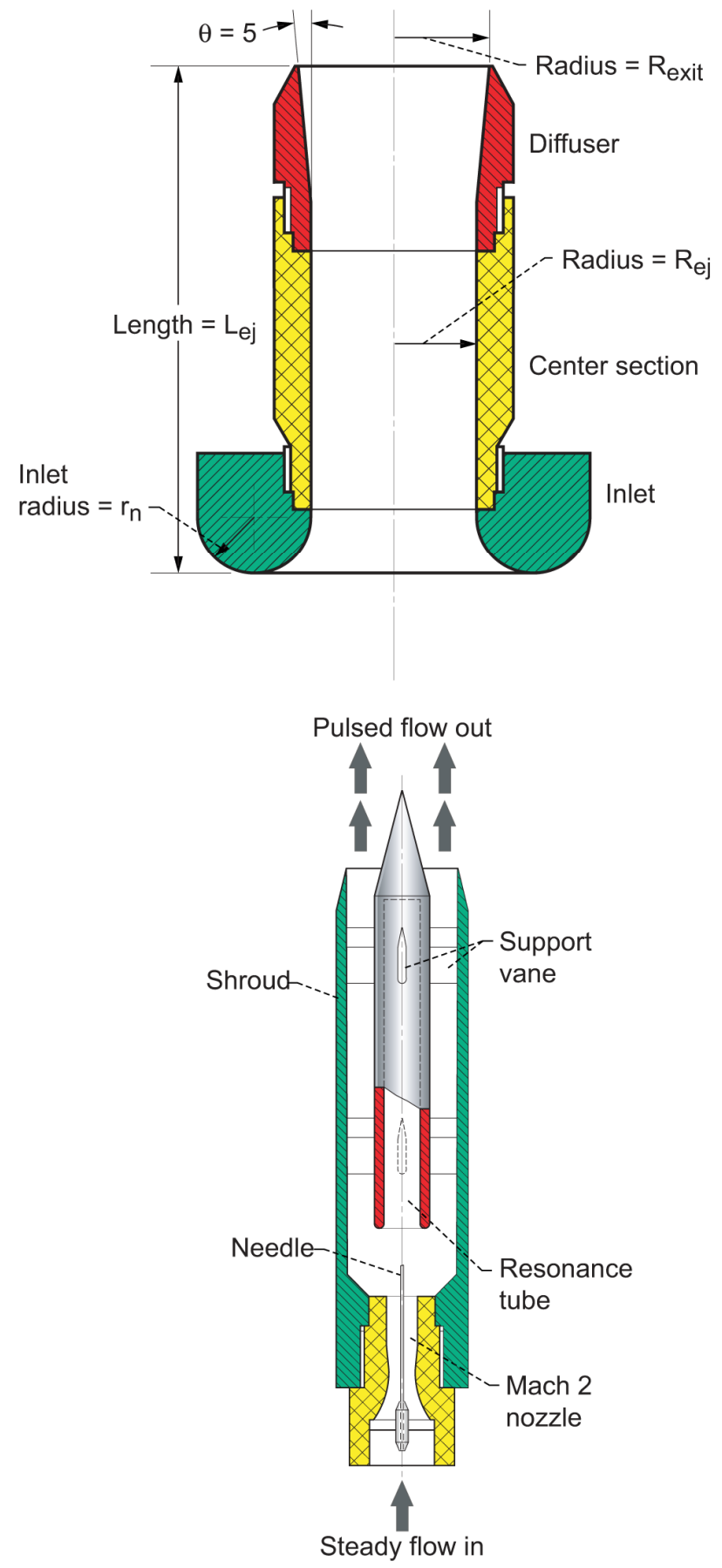

Figure 2. Schematic diagram of the Hartmann-Sprenger tube and the set of ejectors used in the experiment.

\section{Design of the experiment}

The original experimental design of Wilson and Paxson (ref. 8) for a frequency of $550 \mathrm{~Hz}$ was chosen to be a statistical design of experiments, three level, three parameter Box-Behnken scheme (ref. 12), with $L_{e j} / R_{s}, R_{e j} / R_{s,}$ and $r_{n} / R_{S}$ as the three parameters. This does not mean to imply that division by $R_{S}$ is an appropriate scaling; it is simply a convenient way of making the numbers non-dimensional. Initially, the values of $R_{e j} / R_{s}$ were $1.1,1.5$, and 3.0. As the 
thrust augmentations for $R_{e j} / R_{s}=3.0$ were small, it was found useful to add an additional group of ejectors with $R_{e j} / R_{s}=2.0$, using $R_{e j} / R_{s}=1.5$ as the "central" group. In making runs at a frequency of $1100 \mathrm{~Hz}$, it was found that the optimum diameter for thrust augmentation appeared to be shifting towards lower values of $R_{e j} / R_{s}$, and so runs were made for $R_{e j} / R_{s}=1.1,1.5$, and 2.0 only, and not 3.0. As the frequency was lowered, the optimum shifted towards higher $R_{e j} / R_{s}$, and so the values of $R_{e j} / R_{s}$ used were 1.5, 2, and 3, and not 1.1. The full set of runs made is given in table 1 .

TABLE 1.-THE MATRIX OF TEST RUNS COMPRISING THE 3 PARAMETER, 3 LEVEL STATISTICAL EXPERIMENT AT EACH OF THE FOUR FREQUENCIES USED

\begin{tabular}{|c|c|c|c|c|c|c|c|}
\hline \multirow{2}{*}{$\begin{array}{l}\text { Run } \\
\text { No. }\end{array}$} & \multirow[t]{2}{*}{$R_{e j} / R_{s}$} & \multirow[t]{2}{*}{$L_{e j} / R_{S}$} & \multirow[t]{2}{*}{$r_{n} / R_{s}$} & \multicolumn{4}{|c|}{$\alpha$} \\
\hline & & & & $f=1100$ & $f=550$ & $f=275$ & $f=125$ \\
\hline 1 & 1.1 & 3.125 & 0.5 & 1.17 & 1.155 & & \\
\hline 2 & 1.1 & 7.125 & 0.25 & 1.149 & 1.16 & & \\
\hline 3 & 1.1 & 7.625 & 0.75 & 1.134 & 1.155 & & \\
\hline 4 & 1.1 & 12.375 & 0.5 & 1.122 & 1.092 & & \\
\hline 5 & 1.5 & 2.875 & 0.25 & 1.099 & 1.264 & & \\
\hline 6 & 1.5 & 3.375 & 0.75 & 1.053 & 1.275 & & \\
\hline 7 & 1.5 & 7.375 & 0.5 & 1.172 & 1.330 & & \\
\hline 8 & 1.5 & 7.375 & 0.5 & 1.167 & 1.325 & & \\
\hline 9 & 1.5 & 7.375 & 0.5 & 1.169 & 1.308 & & \\
\hline 10 & 1.5 & 12.125 & 0.25 & 1.166 & 1.255 & & \\
\hline 11 & 1.5 & 12.625 & 0.75 & 1.165 & 1.267 & & \\
\hline 12 & 2 & 3.125 & 0.5 & 1.022 & 1.118 & & \\
\hline 13 & 2 & 7.125 & 0.25 & 1.092 & 1.206 & & \\
\hline 14 & 2 & 7.625 & 0.75 & 1.10 & 1.226 & & \\
\hline 15 & 2 & 12.375 & 0.5 & 1.167 & 1.266 & & \\
\hline 16 & 3 & 3.125 & 0.5 & & 1.034 & & \\
\hline 17 & 3 & 7.125 & 0.25 & & 1.075 & & \\
\hline 18 & 3 & 7.625 & 0.75 & & 1.085 & & \\
\hline 19 & 3 & 12.375 & 0.5 & & 1.139 & & \\
\hline 20 & 1.5 & 3.125 & 0.5 & & & 1.287 & 1.14 \\
\hline 21 & 1.5 & 7.125 & 0.25 & & & 1.320 & 1.221 \\
\hline 22 & 1.5 & 7.625 & 0.75 & & & 1.355 & 1.237 \\
\hline 23 & 1.5 & 12.375 & 0.5 & & & 1.326 & 1.265 \\
\hline 24 & 2 & 2.875 & 0.25 & & & 1.277 & 1.186 \\
\hline 25 & 2 & 3.375 & 0.75 & & & 1.343 & 1.218 \\
\hline 26 & 2 & 7.375 & 0.5 & & & 1.379 & 1.317 \\
\hline 27 & 2 & 7.375 & 0.5 & & & 1.373 & 1.295 \\
\hline 28 & 2 & 7.375 & 0.5 & & & 1.376 & 1.313 \\
\hline 29 & 2 & 12.125 & 0.25 & & & 1.305 & 1.271 \\
\hline 30 & 2 & 12.625 & 0.75 & & & 1.383 & 1.310 \\
\hline 31 & 3 & 3.125 & 0.5 & & & 1.053 & 1.075 \\
\hline 32 & 3 & 7.125 & 0.25 & & & 1.106 & 1.137 \\
\hline 33 & 3 & 7.625 & 0.75 & & & 1.116 & 1.182 \\
\hline 34 & 3 & 12.375 & 0.5 & & & 1.197 & 1.250 \\
\hline
\end{tabular}

\section{Experimental results}

The measurements of thrust augmentation found in the various sets of Box-Behnken runs are given in table 1 and in figures 3 (a) to (d), in which thrust augmentation is plotted against normalized ejector length, $L_{e j} / R_{s}$, for each normalized ejector diameter, $R_{e j} / R_{s}$, at different values of the normalized nose radius, $r_{n} / R_{s}$. The 90 percent confidence level in $\alpha$ is \pm 0.03 . The data from a Box-Behnken three parameter set can be fitted with a response surface of the form 

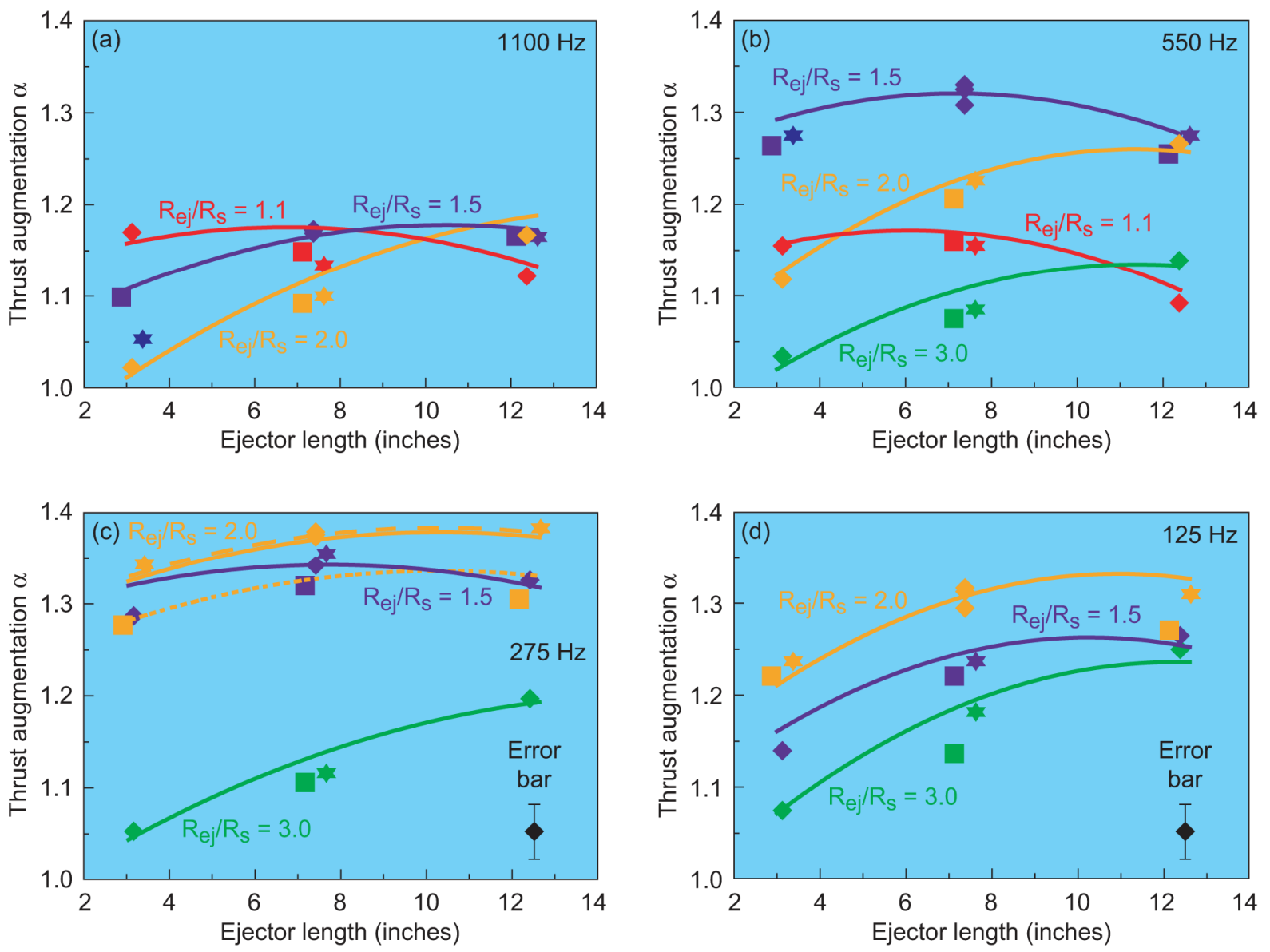

Figure 3. Results from the statistical experiments at four different frequencies. (a) $1100 \mathrm{~Hz}$. (b) $550 \mathrm{~Hz}$. (c) $275 \mathrm{~Hz}$. (d) $125 \mathrm{~Hz}$. Different symbols are used for each nose radius, $\square, r_{n} / \mathbf{R}_{s}=0.25 ; \bullet, r_{n} / R_{s}=0.50$; $\star, r_{n} / R_{s}=0.75$. Different colors are used for each ejector radius, red, $R_{e j} / R_{s}=1.1 ;$ dark blue, $R_{e j} / R_{s}=1.5$; orange, $R_{\mathrm{ej}} / \mathbf{R}_{\mathrm{s}}=2.0$; green, $\mathbf{R}_{\mathrm{ej}} / \mathbf{R}_{\mathrm{s}}=\mathbf{3 . 0}$. The points are experimental data, the lines are response surface fits: dotted, $r_{n} / R_{s}=0.25$; solid, $r_{n} / R_{s}=0.50 ;$ dashed, $r_{n} / R_{s}=0.75$.

$$
\begin{array}{rlll}
\alpha=b_{0} & +b_{1} \cdot\left(L_{e j} / R_{S}\right) & +b_{2} \cdot\left(R_{e j} / R_{S}\right) & +b_{3} \cdot\left(r_{n} / R_{S}\right) \\
& +b_{11} \cdot\left(L_{e j} / R_{S}\right)^{2} & +b_{22} \cdot\left(R_{e j} / R_{s}\right)^{2} & +b_{33} \cdot\left(r_{n} / R_{s}\right)^{2} \\
& +b_{12} \cdot\left(L_{e j} / R_{S}\right) \cdot\left(R_{e j} / R_{S}\right) & +b_{13} \cdot\left(L_{e j} / R_{S}\right) \cdot\left(r_{n} / R_{S}\right) & +b_{23} \cdot\left(R_{e j} / R_{S}\right) \cdot\left(r_{n} / R_{S}\right)
\end{array}
$$

where the values of the constants $b_{i j}$ are determined from the data. These were found by inserting the data into a computer program (Seshadri and Demming (ref. 13)), which calculates the constants, and values of the confidence level for each constant. Constants with low confidence level were eliminated, until only terms with levels greater than 90 percent were retained. The resulting constants are listed in table 2 . Once the response surface is known, the optimum thrust augmentation can be predicted, together with the values of $L_{e j} / R_{s}, R_{e j} / R_{s}$, and $r_{n} / R_{s}$ at which the optimum occurs. These values are also listed in table 2 . 
TABLE 2.-CONSTANTS FOR EQUATION (1)—THE RESPONSE SURFACE OF THE BOX-BEHNKEN EXPERIMENTAL DESIGN, TOGETHER WITH PREDICTIONS FOR THE MAXIMUM THRUST AUGMENTATION AT EACH FREQUENCY.

\begin{tabular}{lcccc} 
& $f=1100 \mathrm{~Hz}$ & $f=550 \mathrm{~Hz}$ & $f=275 \mathrm{~Hz}$ & $f=125 \mathrm{~Hz}$ \\
$\mathrm{~b}_{\mathrm{o}}$ & 1.16812 & -0.18464 & 0.54744 & 0.32863 \\
$\mathrm{~b}_{1}$ & -0.01464 & -0.00713 & - & 0.03207 \\
$\mathrm{~b}_{2}$ & -- & 1.81433 & 0.68699 & 0.64294 \\
$\mathrm{~b}_{3}$ & 0.28836 & 0.23637 & 0.52521 & 0.44842 \\
$\mathrm{~b}_{11}$ & -0.00126 & -0.00197 & -0.00103 & -0.00193 \\
$\mathrm{~b}_{22}$ & -0.07511 & -0.62527 & -0.19356 & -0.15947 \\
$\mathrm{~b}_{33}$ & -0.41306 & -0.21744 & -0.30355 & -0.39603 \\
$\mathrm{~b}_{12}$ & 0.02336 & 0.02591 & 0.01052 & 0.00505 \\
$\mathrm{~b}_{13}$ & 0.01187 & -- & -- & \\
$\mathrm{b}_{23}$ & -- & -- & -0.06310 & 1.338 \\
& & & & 2.21 \\
$\alpha_{\max }$ & 1.189 & 1.331 & 1.385 & 11.2 \\
$\mathrm{R}_{\mathrm{ej}} / \mathrm{R}_{\mathrm{s}}$ & 1.98 & 1.62 & 1.91 & 0.5 \\
$\mathrm{~L}_{\mathrm{ej}} / \mathrm{R}_{\mathrm{s}}$ for $\alpha_{\max }$ & 12.6 & 8.9 & 9.9 & 5.07 \\
$\mathrm{r}_{\mathrm{n}} / \mathrm{R}_{\mathrm{s}}$ & 0.5 & 0.5 & 0.75 & \\
$\mathrm{~L}_{\mathrm{ej}} / \mathrm{R}_{\mathrm{ej}}$ & 6.36 & 5.49 & 5.18 & \\
\hline
\end{tabular}

Although equation (1) includes $r_{n}$, changes in $\alpha$ due to changes in $r_{n}$ for the range of values used are very small, and the changes seen experimentally are statistically insignificant, except at a frequency of $275 \mathrm{~Hz}$ where for $R_{e j} / R_{s}=2$, thrust for $r_{n} / R_{s}=0.25$ seems lower than values for $r_{n} / R_{s}=0.5$, and $r_{n} / R_{s}=0.75$ indicating an optimum value between the latter two values. That, apart from this one case, changes in the nose radius seem unimportant is surprising, and may be a consequence of the limited values of $r_{n}$ used in this experiment. Other experiments (refs. 7 and 15) have shown a significant effect of $r_{n}$, including the possibility of an optimum value. Sections through the response surface at each value of ejector radius, $R_{e j}$, for $r_{n} / R_{s}=0.5$, are also plotted in figure 3 , showing good agreement with the experimental results. The optimum values predicted are little different from the maximum observed experimental values.

\section{Interpretation of the experimental results}

\section{A. Statement of the problem}

It was originally hoped that by finding a response surface at each frequency, some insight would be gained into how the parameters affect the thrust augmentation as a function of the frequency. Except for the conclusion that changes in nose radius were not statistically significant, this was not apparent from the constants listed in table 2. Since the objective here is to find the overall maximum thrust augmentation, and at what values of the parameters this occurs, only the maximum augmentation achieved at any diameter, length and frequency will be considered further. These are listed in table 3. A more detailed look at these data is required.

TABLE 3.- $-L_{p u l s e} / D_{\text {pulse }}$ AND $\varepsilon$ FOR EACH FREQUENCY USED, TOGETHER WITH THE MAXIMUM THRUST AUGMENTATION OBSERVED FOR EACH $R_{e}$, TOGETHER WITH VALUES OF THRUST AUGMENTATION FOR $L_{p u l s e} / D_{p u l s e}=N$, I.E., $\left.\alpha_{v r}\right|_{N}$, AND FOR $L_{p u l s e} / D_{p u l s e}=\infty$, I.E., $\alpha_{\mathrm{ss}}$, AS CALCULATED FROM THE VALUES OF $\alpha$ AT $f=275$, AND $f=125$.

\begin{tabular}{llllcll}
\hline Frequency $(\mathrm{Hz})$ & 1100 & 550 & 275 & 125 & \multicolumn{2}{c}{0} \\
$L_{\text {pulse }} / D_{\text {pulse }}$ & 2.5 & 5 & 10 & 22 & $0.86=N$ & $\infty$ \\
$\varepsilon$ & 0.177 & 0.386 & 0.54 & 0.54 & 0.54 & 0.54 \\
$R / R_{s}$ & 1.2438 & 1.313 & 1.3084 & 1.3084 & 1.3084 & 1.3084 \\
$\alpha_{\max }$ for $R_{e j} / R_{s}=1.1$ & 1.17 & 1.16 & - & - & - & - \\
$\alpha_{\max }$ for $R_{e j} / R_{s}=1.5$ & 1.169 & 1.321 & 1.355 & 1.265 & 1.395 & 1.191 \\
$\alpha_{\max }$ for $R_{e j} / R_{s}=2.0$ & 1.167 & 1.266 & 1.376 & 1.308 & 1.406 & 1.252 \\
$\alpha_{\max }$ for $R_{e j} / R_{s}=3.0$ & - & 1.139 & 1.197 & 1.25 & 1.174 & 1.294 \\
\hline
\end{tabular}

\section{B. Relationship of frequency to $L_{p u l s e} / D_{\text {pulse }}$}

The experiments were performed at four different frequencies. Since the mass flow was constant at all frequencies, the mass per pulse $m_{p u l s e}$ is given by 


$$
m_{\text {pulse }}=\dot{m}_{\text {jet }} / f
$$

But $m_{p}$ is also given by the product of pulse volume and density

$$
m_{\text {pulse }}=\rho \pi D_{\text {pulse }}^{2} L_{\text {pulse }} / 4
$$

from which it is seen that $L_{\text {pulse }} / D_{\text {pulse }}$ is inversely proportional to frequency. In table 3 the values of $L_{\text {pulse }} / D_{\text {pulse }}$ for each frequency used are given. Using the slug model plus some experimental measurements Wilson (ref. 14) has indicated that the value of the formation number is 6.86 for this shrouded Hartmann-Sprenger tube. Thus the two higher frequencies have values of $L_{p u l s e} D_{\text {pulse }}$ below the formation number, whilst the two lower frequencies have values of $L_{\text {pulse }} / D_{\text {pulse }}$ above it.

\section{Thrust augmentation for $L_{\text {pulse }} / D_{\text {pulse }}$ greater than $N$.}

For the measurements at values of $L_{p u l s e} / D_{\text {pulse }}$ above the formation number, the flow consists of a leading vortex ring and a trailing jet. The vortex ring has exactly the same size and properties as that generated at $L_{p u l s e} / D_{\text {pulse }}=N$. The thrust on the ejector will be partially due to the vortex ring and partially to the trailing jet. There will be a time, $t^{\prime}$, following initiation of the jet at which the value of $L_{p u l s e}=\int u(t) \mathrm{d} t$ is equal to N.D pulse. The fraction of the total jet impulse that is in this portion of the jet is

$$
p=\int_{o}^{t^{\prime}} u(t)^{2} \mathrm{~d} t / \int_{o}^{\tau} u(t)^{2} \mathrm{~d} t
$$

It is this fraction of the impulse that goes into forming the vortex ring, giving rise to a thrust augmentation $\alpha_{v r}$. The remaining impulse will be in the trailing jet, which will be assumed to create a thrust augmentation $\alpha_{s s}$ as would a steady jet. Thus, for $L_{p u l s e} / D_{\text {pulse }}$ greater than the formation number, the total thrust augmentation will be

$$
\alpha_{\text {total }}=p \alpha_{v r}+(1-p) \alpha_{s s}
$$

in which $\alpha_{v r}$ is calculated at the formation number, i.e., it is $\left.\alpha_{v r}\right|_{N}$. A measurement of the jet exit velocity $u(t)$ for this tube has been reported previously (Wilson (ref. 14)). Given this distribution, the integrals which determine $p$ can be performed numerically. The result is given in table 4. Of course, if $L_{p u l s e} / D_{p u l s e}<N$, then $\alpha_{\text {total }}=\alpha_{v r}$, with no contribution from $\alpha_{s s}$.

TABLE 4.-VALUES OF $p$ AS A FUNCTION OF $L_{\text {pulse }} / D_{\text {pulse }}$ FOR THE JET VELOCITY OF THE HARTMANN-SPRENGER TUBE

\begin{tabular}{lcccccccccccc}
\hline$L_{\text {pulse }} / D_{\text {pulse }}$ & 6.86 & 7.4 & 8 & 10 & 12 & 14 & 16 & 18 & 20 & 22 & 24 & $\infty$ \\
$p=$ & 1 & 0.9736 & 0.9424 & 0.8064 & 0.6724 & 0.5742 & 0.5002 & 0.4443 & 0.3990 & 0.3649 & 0.3363 & 0 \\
\hline
\end{tabular}

At each of the two low frequencies, the value of $p$ is known from table 4, i.e., $p=0.8064$ at $L_{\text {pulse }} / D_{\text {pulse }}=10$, and 0.3649 at $L_{\text {pulse }} / D_{\text {pulse }}=22$. At each frequency, there are three values of $\alpha_{\text {total }}$, one for each different ejector radius. Thus for each radius, there are two values of $\alpha_{\text {total }}$, which can be substituted into an equation (5) for each frequency, so that the resulting pair of equations can be solved for $\left.\alpha_{v r}\right|_{N}$, and $\alpha_{s s}$. Values of $\left.\alpha_{v r}\right|_{N}$, and $\alpha_{s s}$ are given in table 3 . The values of $\alpha_{s s}$ for each ejector radius are fitted by the equation:

$$
\alpha_{s s}=1.094+0.158 \ln \left(2 R_{\text {exit }} / D_{\text {pulse }}\right)
$$

As can be seen, $\alpha_{s s}$ increases as the ratio of ejector exit diameter to tube diameter increases. This trend, and the values of the thrust augmentation are in agreement with values of steady state thrust augmentation given by Porter and Squyers (ref. 16).

\section{Thrust augmentation for $L_{\text {pulse }} / D_{\text {pulse }}$ less than $N$}

The objective of this work is to gain some understanding of the conditions which lead to maximum thrust augmentation, in particular ejector radius, and vortex ring size. Length is of course also an important parameter, but the value of $L_{\text {ejector }} / R_{\text {ejector }}$ at $\alpha_{\max }$, also given in table 2, and plotted in figure 4, does not appear to be very dependent 
on $L_{\text {pulse }} / D_{\text {pulse. At }} L_{\text {pulse }} / D_{\text {pulse }}=N$, the value of $L_{\text {ejector }} / R_{\text {ejector }}$ for $\alpha_{\text {max }}$ is $5.2-5.5$, and since from figure 3, at the maximum value of thrust augmentation for any of the curves, the augmentation does not depend strongly on ejector length, a value of $L_{\text {ejector }} / R_{\text {ejector }}=5.3$ would probably be reasonably close to optimum at any frequency. To investigate the effects of ejector radius and vortex ring size, it was decided to use the values of maximum thrust augmentation observed at the two higher frequencies, together with the values calculated for $\left.\alpha_{v r}\right|_{N}$ from the low frequency data, as the dependant parameter in creating a response surface in parameter space. A major question was what are the appropriate independent parameters to use in forming a response surface. Clearly, the thrust augmentation depends on the vortex ring properties, and the ejector size. Although $L_{\text {pulse }} / D_{\text {pulse }}$ is important for vortex ring formation, once the ring is formed, the ratio $\varepsilon$ is what characterizes it, and was chosen as the parameter for the vortex ring. For the ejector, the choice was not so clear. Considering the thrust augmentation, if the total exit flow from the ejector can be described by an average velocity in time and radius, $V_{\text {exit }}$, and the jet has an average velocity $U$ such that $T_{j e t}=\dot{m}_{j e t} U$, then:

$$
\begin{aligned}
\alpha & =\text { total thrust } / \text { thrust of jet alone } \\
& =\dot{m}_{\text {total }} V_{\text {exit }} / \dot{m}_{\text {jet }} U \\
& =\rho \pi\left(R_{\text {exit }} V_{\text {exit }}\right)^{2} / \rho \pi \xi\left(D_{\text {pulse }} U / 2\right)^{2} \\
& =\left(2 R_{\text {exit }} / D_{\text {pulse }}\right)^{2}\left(V_{\text {exit }} / U\right)^{2} / \xi
\end{aligned}
$$

from which:

$$
V_{\text {exit }} / U=\sqrt{\alpha \xi}\left(D_{\text {pulse }} / 2 R_{\text {exit }}\right)
$$

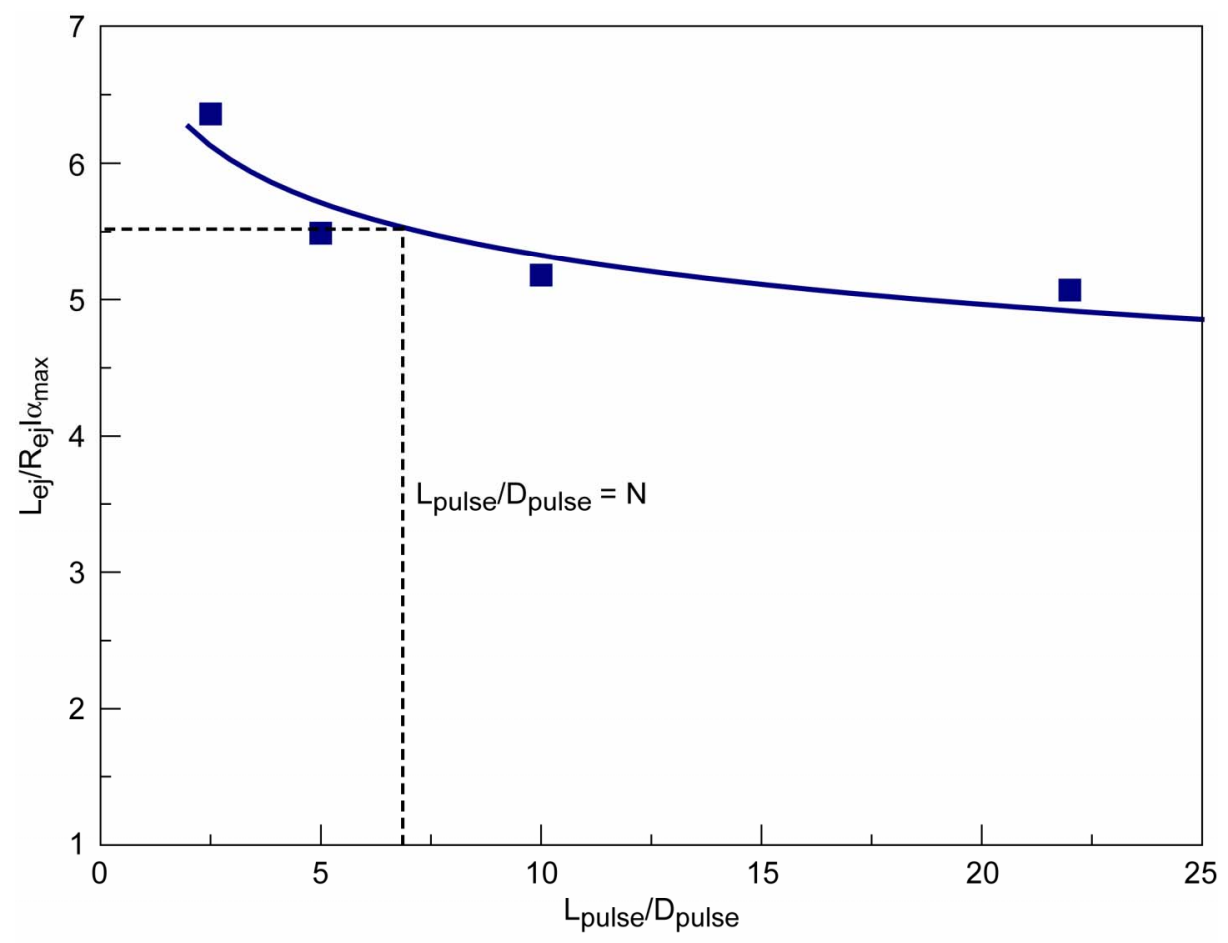

Figure 4. $L_{e j} / R_{e j}$ at $\alpha_{\max }$ versus $L_{\text {pulse }} / D_{\text {pulse }}$. 


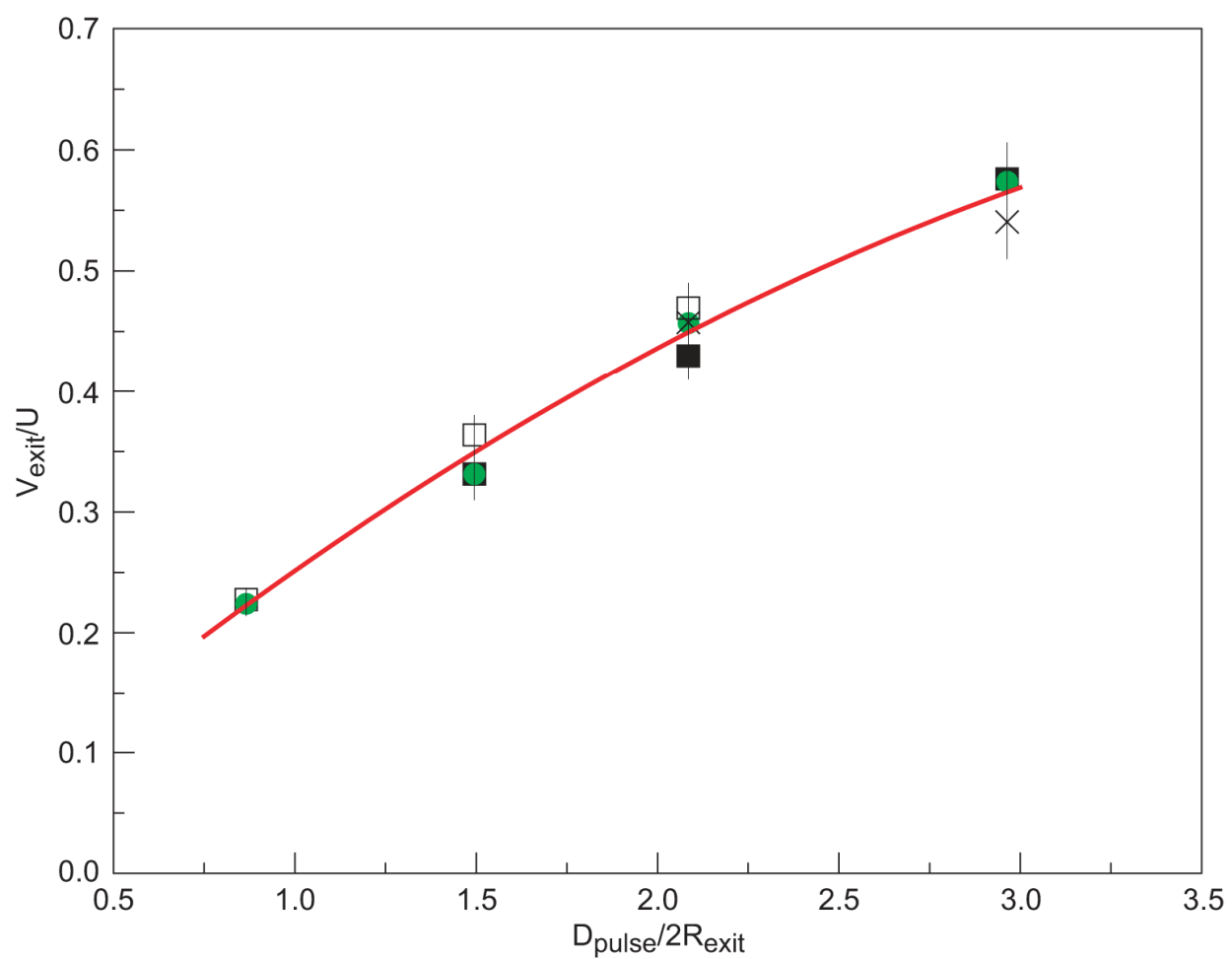

Figure 5. $V_{\text {exit }} / \mathrm{U}$ versus $D_{\text {pulse }} / 2 R_{\text {exit }} \cdot \mathbf{L}_{\text {pulse }} / \mathrm{D}_{\text {pulse }}=2.5, \bullet \mathrm{L}_{\text {pulse }} / \mathrm{D}_{\text {pulse }}=5$, $\square \mathrm{L}_{\text {pulse }} / \mathrm{D}_{\text {pulse }}=6.86$, calculated from measurements of $\alpha, \times \mathrm{L}_{\text {pulse }} / \mathrm{D}_{\text {pulse }}=5$, from direct measurement of $\mathrm{V}_{\text {exit }} / \mathrm{U}$.

Values of $V_{\text {exit }} / U$ derived from equation (8) are plotted against $D_{\text {pulse }} / 2 R_{\text {exit }}$ for the points in table 3 with $L_{\text {pulse }} / D_{\text {pulse }}$ less than or equal to $N$ in figure 5, as well as two measured values of $V_{\text {exit }} / U$ reported by Wilson and Paxson (ref. 8). Within the experimental error, these points are all fitted by a single line, shown in the figure. The equation of the line is:

$$
V_{\text {exit }} / U=-0.123+1.442 D_{\text {pulse }} / 2 R_{\text {exit }}-0.648\left(D_{\text {pulse }} / 2 R_{\text {exit }}\right)^{2}
$$

This suggests that $D_{\text {pulse }} / 2 R_{\text {exit }}$ would be an appropriate parameter for fitting $V_{\text {exit }} / U$ to the ejector size, from which the thrust augmentation could be obtained. Unfortunately, this did not prove to be the case, since small errors in fitting $V_{\text {exit }} / U$ are magnified in calculating thrust augmentation, as it is the square of $V_{\text {exit }} / U$ that appears in the equation for thrust augmentation. Nevertheless, the fit to $V_{\text {exit }} / U$ is useful in calculating an estimate of the mass entrainment, since, as can be shown easily:

$$
\alpha=(1+\beta) V_{\text {exit }} / U
$$

Instead of using the fit to $V_{\text {exit }} / U$, all the values of thrust augmentation given in table 3 were entered into a nonlinear regression analysis with $\varepsilon$ and one of either $D_{\text {pulse }} / 2 R_{\text {exit }}, R / R_{\text {exit }}$, or $(R+a) / R_{\text {exit }}$ as the independent parameters. It was found that the best fit was with $(R+a) / R_{\text {exit }}$ as the second parameter, with the resulting fit being:

$$
\begin{aligned}
\alpha & =1.816-3.743 \varepsilon+0.0389 \varepsilon^{2}-1.447(R+a) / R_{\text {exit }}+0.679\left((R+a) / R_{\text {exit }}\right)^{2} \\
& +8.431 \varepsilon(R+a) / R_{\text {exit }}-4.014 \varepsilon\left((R+a) / R_{\text {exit }}\right)^{2}
\end{aligned}
$$

Using this fit, together with thrust augmentation calculated from equation (5) for $L_{\text {pulse }} / D_{\text {pulse }}$ greater than $N$, thrust augmentation is plotted against ejector radius in figure 6, and against $L_{p u l s e} / D_{p u l s e}$ in figure 7. In using this fit, 
the values of $R$ and $\varepsilon$ (and hence $a$ ) as a function of $L_{p u l s e} / D_{p u l s e}$ were derived from slug theory as described by Wilson (ref. 14), $R / R_{s}$ and $\varepsilon$ are given in table 3 .

\section{Discussion}

The plot of thrust augmentation versus ejector radius in figure 6 shows that the optimum ejector radius increases as $L_{p u l s e} / D_{\text {pulse }}$ increases, as had already been observed in figure 2 . The optimum ejector radius for $L_{p u l s e} / D_{\text {pulse }}=N$ is at $R_{e j} / R_{s}=1.75$, which corresponds to $R_{e j} /(R+a)=0.87$, i.e., the optimum ejector is slightly smaller than the vortex ring size. The plot of thrust augmentation against $L_{p u l s e} / D_{\text {pulse }}$ in figure 7 shows that there is a peak thrust augmentation at $L_{\text {pulse }} / D_{\text {pulse }}=N$. However, as $L_{p u l s e} / D_{\text {pulse }}$ gets very much larger than $N$, the thrust augmentation approaches $\alpha_{s s}$, which increases monotonically with $R_{\text {exit }}$, so there will no longer be an optimum ejector radius at very large $L_{\text {pulse }} / D_{\text {pulse }}$. The optimum thrust augmentation at $L_{p u l s e} / D_{\text {pulse }}=N$ is around 1.4, considerably lower than the values around 2.0 reported by Lockwood (ref. 4), Didelle (ref. 5), and Paxson, Wilson, and Dougherty (ref. 7). It has been shown (Wilson (ref. 14)) that the circulation produced by the annular exit of the shrouded HartmannSprenger tube $\left(K=13.7 \mathrm{~m}^{2} / \mathrm{s}\right)$ is lower than would be expected for the same value of $U$ from a circular exit $(K=20$ $\mathrm{m}^{2} / \mathrm{s}$ ). Since the vortex ring velocity is proportional to the circulation, a circular exit would have a higher value of vortex ring velocity, and hence, presumably, also a higher value of $V_{\text {exit. }}$ If it is also assumed that the thrust augmentation is proportional to $V_{\text {exit }} U$, then a circular tube producing the same exit velocity as the HartmannSprenger tube could be expected to generate a thrust augmentation of 2.04, in line with the values from the other experiments. However, equation (7) shows thrust augmentation proportional to $\left(V_{\text {exit }} / U\right)^{2}$, which would suggest that a maximum $\alpha_{v r}$ of 3 should be possible.

These experiments indicate that a length of about two and a half times the ejector diameter is optimum. This is longer than claimed by Lockwood (ref. 4), but shorter than optimum length to diameter ratios found by Paxson, Wilson and Dougherty (ref. 7), or Didelle (ref. 5), The results given in table 2 for the optimum ejector length to diameter ratio seem to show a gradual decrease in the ratio as $L_{\text {pulse }} / D_{\text {pulse }}$ increases. Thus the discrepancy in optimum ejector length to diameter between this work and those of Lockwood, and Paxson, Wilson and Dougherty might be explained as due to being in very different regimes of $L_{p u l s e} / D_{\text {pulse }}$. Didelle's maximum thrust augmentation was at a Strouhal number of 0.07 . Because his valve gave two pulses per second, the value of $L_{\text {pulse }} / D_{\text {pulse }}$ used by Didelle is $1 / 2 S_{t}$, i.e., 7 at maximum thrust augmentation. Most of his runs were for $L_{p u l s e} / D_{\text {pulse }}<7$, with a few at $L_{p u l s e} / D_{\text {pulse }}=10$. It is gratifying that Didelle's optimum value of $L_{p u l s e} / D_{\text {pulse }}$ is in close agreement with that found here, but that leaves the discrepancy of the optimum ejector length to diameter ratio unexplained.

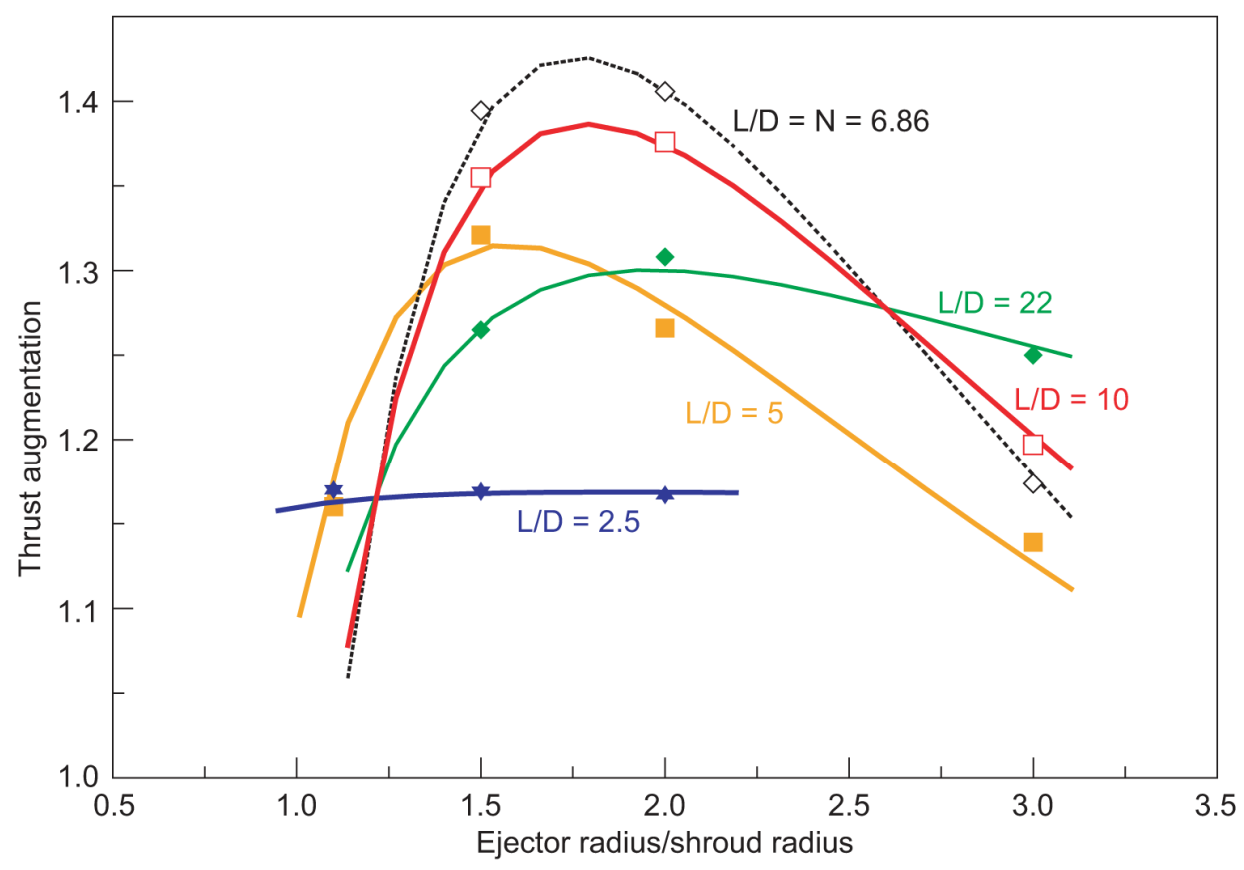

Figure 6. Thrust augmentation versus $\mathbf{R}_{\mathrm{ej}} / \mathbf{R}_{\mathbf{s}}$. 


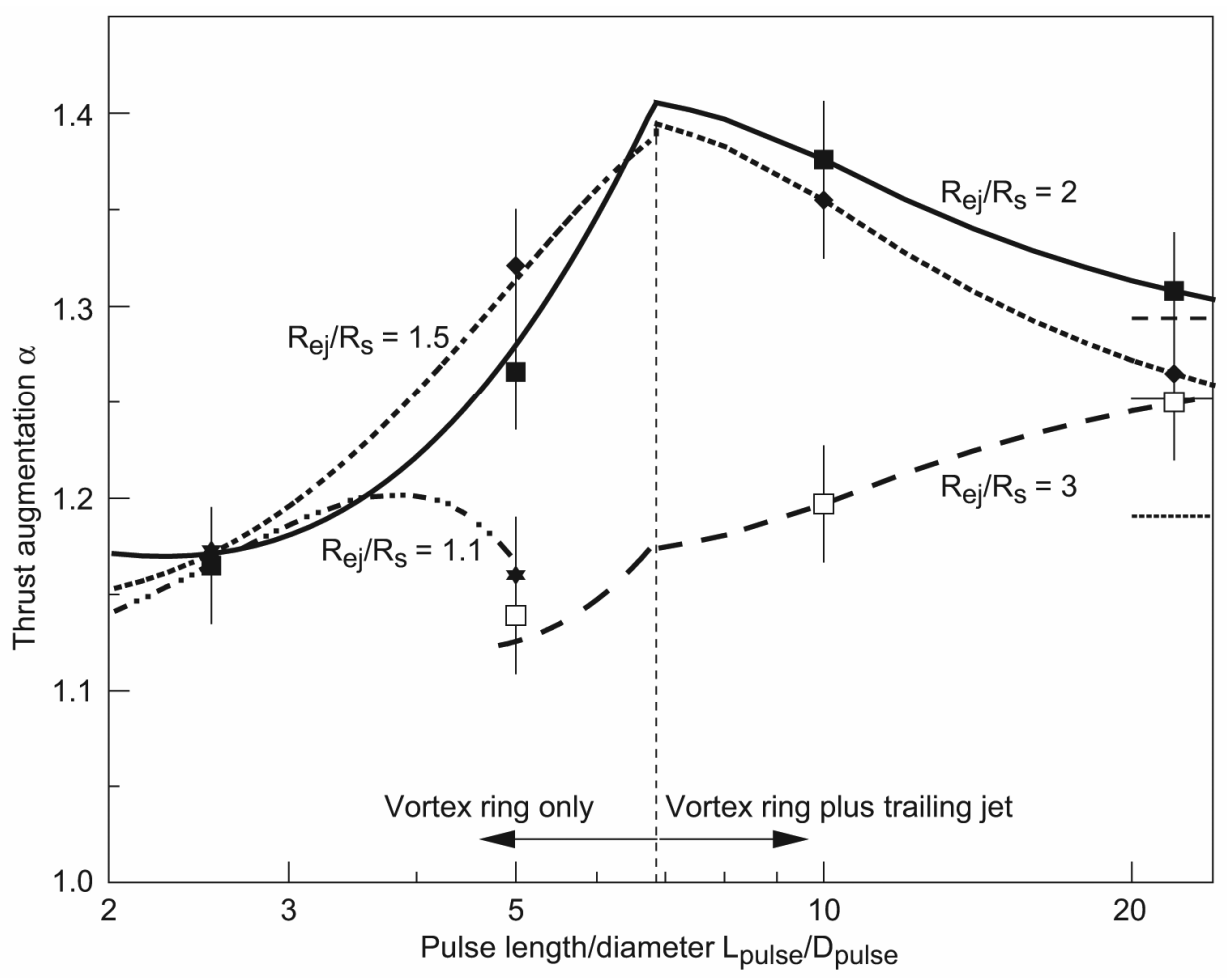

Figure 7. Thrust augmentation versus $L_{\text {pulse }} / D_{\text {pulse }}$ for each value of $R_{e j} / R_{s}$. The thin horizontal lines at the right represent the values of $\alpha_{s s}$. The data and fitted lines correspond to different values of $R_{\mathrm{ej}} / \mathbf{R}_{\mathrm{s}}$, namely: $\mathbf{R}_{\mathrm{ej}} / \mathbf{R}_{\mathrm{s}}=1.1, \ldots \cdots$, $\mathbf{R}_{\mathrm{ej}} / \mathbf{R}_{\mathrm{s}}=1.5, \cdots, \bullet, \mathbf{R}_{\mathrm{ej}} / \mathbf{R}_{\mathrm{s}}=2, \square, \mathbf{}, \mathbf{R}_{\mathrm{ej}} / \mathbf{R}_{\mathrm{s}}=3,---, \square$.

In these experiments, Didelle's experiments, and the experiments with pulsejet drivers, the length of the pulse increases with the inverse of the frequency, and so low frequency approaches steady state flow. Both Didelle (ref. 5), and Wilson and Paxson (ref. 8) found that $V_{\text {exit }}$ had a large average component, with an oscillation on top of it, such that the ejector flow never reached zero velocity. Care must be used in relating the results to pulse detonation devices, where the pulse length is determined by the length of the tube, and is independent of frequency. Because the duty cycle is very low in detonation devices, $V_{\text {exit }}$ may drop to zero between pulses. This is less likely as the frequency increases, and so high frequency is more likely to approach a steady state flow in the ejector.

\section{Conclusions}

In conclusion, for the Hartmann-Sprenger tube investigated, unsteady thrust augmentation is shown to have a peak when the driving pulse jet has a pulse length to diameter ratio equal to the formation number. For values of $L_{p u l s e} / D_{\text {pulse }}$ close to $N$, the optimum ejector radius will be equal to $0.87(R+a)$. As $L_{p u l s e} / D_{\text {pulse }}$ increases, the optimum ejector radius also increases, until for very large values of $L_{p u l s e} / D_{p u l s e}$, the thrust augmentation approaches steady-state values, increasing monotonically with ejector radius. The optimum ejector length appears to be about 2.5 times the ejector diameter, although this does not agree with the results of other workers (refs. 4, 5, and 7).

\section{References}

1. R. Shehadeh, S. Saretto, S.-Y. Lee, S. Pal, and R.J. Santoro "Thrust Augmentation Experiments for a Pulse Detonation Driven Ejector," Paper AIAA-2004-3398, presented at the $40^{\text {th }}$ AIAA/ASME/SAE/ASEE Joint Propulsion Conference, Fort Lauderdale, FL (2004).

2. Allgood, E. Gutmark, J. Hoke, R. Bradley, and F. Schauer "Performance Measurements of Pulse Detonation Engine Ejectors," Paper AIAA-2004-3398, presented at the $40^{\text {th }}$ AIAA/ASME/SAE/ASEE Joint Propulsion Conference, Fort Lauderdale, FL (2004). 
3. Rasheed, V. Tangirala, P.F. Pinard, and A.J. Dean, "Experimental and Numerical Investigations of Ejectors for PDE Applications," Paper AIAA-2003-4971, presented at the $39^{\text {th }}$ AIAA/ASME/SAE/ASEE Joint Propulsion Conference, Huntsville, AL (2003).

4. Lockwood, R.M. "Interim Summary Report on Investigation of the Process of Energy Transfer from an Intermittent Jet to Secondary Fluid in an Ejector-type Thrust Augmenter," Hiller Aircraft Report No. ARD-286, 1961.

5. Didelle, H. "L'augmentation de Poussée des trompes à jets pulsants ou battants," Doctor of Engineering thesis presented to the Scientific and Medical University and the National Polytechnical Institute of Grenoble, France 1976.

6. Bertin, J. "Dilution pulsatoire sur réacteur," Comptes Rendues des Séances de l'Académie des Sciences, vol. 240, pp. 1855-1857, 1955.

7. Paxson, D.E., Wilson, J., and Dougherty, K.T. "Unsteady Ejector Performance: An Experimental Investigation using a Pulsejet Driver," Paper AIAA-2002-3915, presented at the $38^{\text {th }}$ Joint AIAA/ASME/SAE/ASEE Propulsion Conference, Indianapolis, IN, 2002.

8. Wilson, J., and Paxson, D.E. "Unsteady Ejector Performance: An Experimental Investigation using a Resonance Tube Driver," Paper AIAA-2002-3632, presented at the $38^{\text {th }}$ AIAA/ASME/SAE/ASEE Joint Propulsion Conference, Indianapolis, IN, 2002.

9. Elder, F.K. and de Haas, N. "Experimental Study of the Formation of a Vortex Ring at the Open End of a Cylindrical Shock Tube," Journal of Applied Physics, 23, pp. 1065-1069, 1952.

10. Das, D., Arakeri, J.H., Krothapalli, A., and Lourenco, L.M. "Compressible Vortex Ring: A PIV Study" Paper AIAA-2001-2214, presented at the $7^{\text {th }}$ AIAA/CEAS Aeroacoustics Conference, Maastrich, Netherlands, 2001.

11. Gharib, M., Rambod, E., and Shariff, K. “A universal time scale for vortex ring formation,” J. Fluid Mech., 360, pp. 121-140, 1998.

12. Mason, R.L. Gunst, R.F. and Hess, J.L. "Statistical Design and Analysis of Experiments: with Applications to Engineering and Science," Wiley, New York, 1989.

13. Seshadri, S. and Demming, S.N. "Box-B Interactive Computer Programs for using Three-and Four-Factor BoxBehnken Designs in Research, Development, and Manufacturing (version 2.1)," This program is available from: Statistical Programs, 9941 Rowlett, Suite 6, Houston, TX 77075.

14. Wilson, J. "Vortex Rings generated by a Shrouded Hartmann-Sprenger Tube," Paper AIAA-2005-5163, presented at the $35^{\text {th }}$ AIAA Fluid Dynamics Conference, Toronto, Canada, June 2005.

15. Wilson, J., Sgondea, A., Paxson, D.E., and Rosenthal, B.N. "Parametric Investigation of Thrust Augmentation by Ejectors on a Pulsed Detonation Tube," Paper AIAA-2005-4208, presented at the $41^{\text {st }}$ AIAA/ASME/SAE/ASEE Joint Propulsion Conference, Tucson, AZ, 2005.

16. Porter, J.L., and Squyers, R.A. "A Summary/Overview of Ejector Augmentor Theory and Performance, Phase II-Technical Report," Vought Corporation Advanced Technology Center Report No. R-91100/9CR-47A., 1979. 


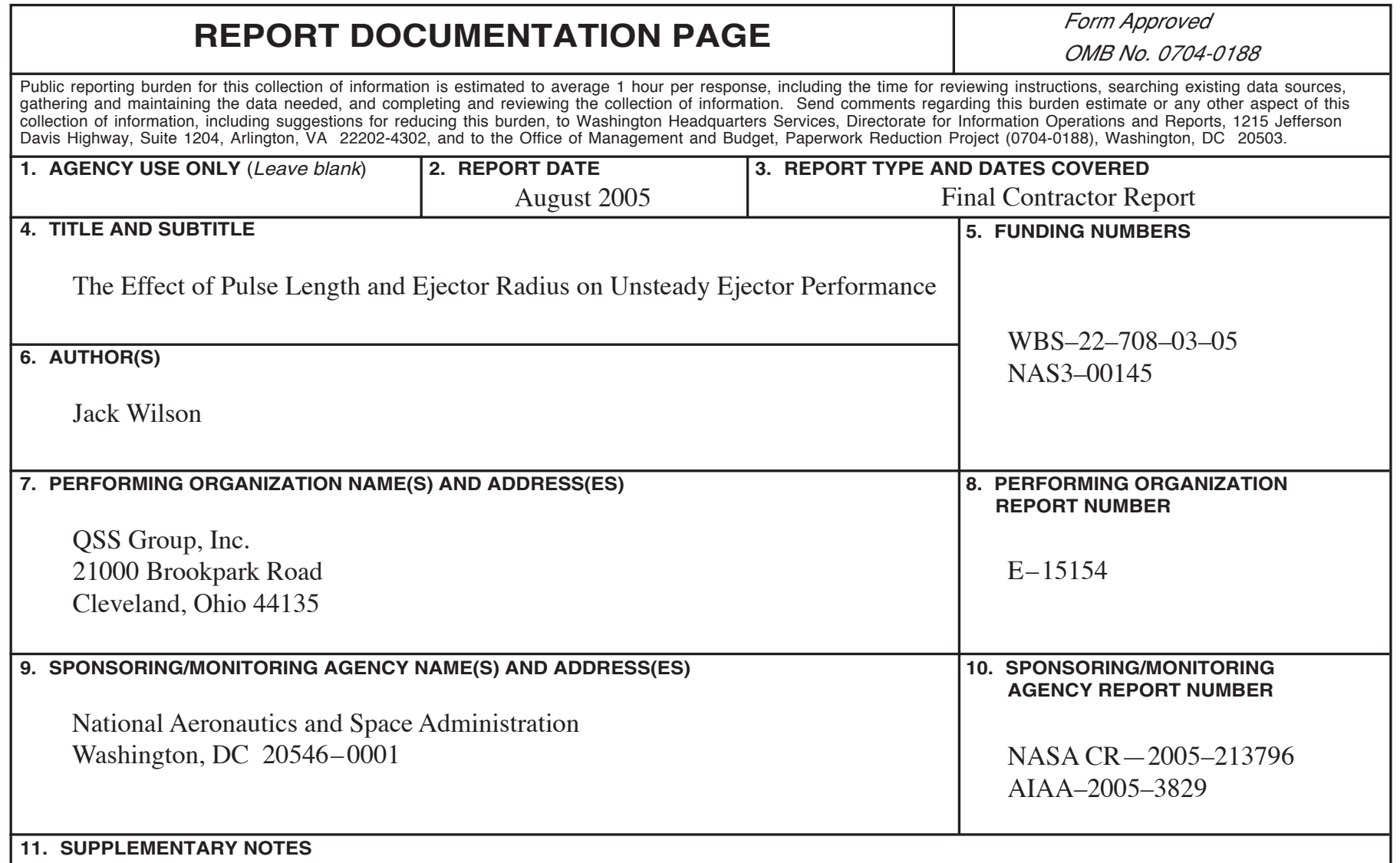

Prepared for the 41st Joint Propulsion Conference and Exhibit cosponsored by the AIAA, ASME, SAE, and ASEE, Tucson, Arizona, July 10-13, 2005. Project Manager, Richard L. DeLoof, Aeronautics Division, NASA Glenn Research Center, organization code PRV, 216-433-6632.

\begin{tabular}{|l|l|l|l|l}
\hline 12a. DISTRIBUTION/AVAILABILITY STATEMENT DISTRIBUTION CODE & 12b. D
\end{tabular}

Unclassified - Unlimited

Subject Categories: 02 and 07

Available electronically at http://gltrs.grc.nasa.gov

This publication is available from the NASA Center for AeroSpace Information, 301-621-0390.

13. ABSTRACT (Maximum 200 words)

The thrust augmentation of a set of ejectors driven by a shrouded Hartmann-Sprenger tube has been measured at four different frequencies. Each frequency corresponded to a different length to diameter ratio of the pulse of air leaving the driver shroud. Two of the frequencies had length to diameter ratios below the formation number, and two above. The formation number is the value of length to diameter ratio below which the pulse converts to a vortex ring only, and above which the pulse becomes a vortex ring plus a trailing jet. A three level, three parameter Box-Behnken statistical design of experiment scheme was performed at each frequency, measuring the thrust augmentation generated by the appropriate ejectors from the set. The three parameters were ejector length, radius, and inlet radius. The results showed that there is an optimum ejector radius and length at each frequency. Using a polynomial fit to the data, the results were interpolated to different ejector radii and pulse length to diameter ratios. This showed that a peak in thrust augmentation occurs when the pulse length to diameter ratio equals the formation number, and that the optimum ejector radius is 0.87 times the sum of the vortex ring radius and the core radius.

14. SUBJECT TERMS

\begin{tabular}{|l|l|}
\hline & $\begin{array}{c}15 . \text { NUMBER OF PAGES } \\
19\end{array}$ \\
\cline { 2 - 2 } & 16. PRICE CODE \\
\hline $\begin{array}{c}\text { 19. SECURITY CLASSIFICATION } \\
\text { OF ABSTRACT } \\
\text { Unclassified }\end{array}$ & 20. LIMITATION OF ABSTRACT \\
\hline
\end{tabular}

Ejectors; Thrust augmentation; Vortex rings

Standard Form 298 (Rev. 2-89)

NSN 7540-01-280-5500 

